Check for updates

Cite this: RSC Adv., 2019, 9, 31474

Received 15th August 2019

Accepted 26th September 2019

DOI: $10.1039 / c 9 r a 06368 d$

rsc.li/rsc-advances

\section{Combinatorial synthesis and biological evaluations of $(E)$ - $\beta$-trifluoromethyl vinylsulfones as antitumor agents $\dagger$}

\author{
Haosha Tang, ${ }^{\text {a }}$ Yunyan Kuang, ${ }^{\mathrm{b}}$ Julan Zeng, ${ }^{\mathrm{c}}$ Xiaofang Li, ${ }^{\text {d }}$ Wei Zhou (D) *b \\ and Yuan Lu*a
}

Combinatorial synthesis of $(E)$ - $\beta$-trifluoromethyl vinylsulfones is accomplished through a reaction of alkynes, Togni reagent, and sodium benzenesulfinates in DMSO under metal-free conditions at room temperature. These compounds are evaluated in several assays against different tumor cells. Some hits are identified against ES-2, HO-8910, and K562.

\section{Introduction}

The chalcogen-containing skeleton has become a privileged and attractive scaffold in medicinal chemistry owing to its unique biological activities. ${ }^{1}$ In particular, more and more novel biological effects of vinyl sulfone compounds have been discovered recently. It has been reported that structures containing an $\alpha, \beta$ unsaturated vinylsulfone moiety exhibit modest inhibitory potencies in inflammation as a novel class toward Parkinson' $\mathrm{s}^{2,3}$ and arthritis ${ }^{4}$ disease therapy by depressing the expression of endothelial cells of adhesion molecules, including vascular cell adhesion molecule-1 (VCAM-1) and intercellular adhesion molecule-1 (ICAM-1), as well as inhibiting the nuclear factor E2related factor 2 (Nrf2) pathway which is responsible for the cellular defense system against oxidative stress. They also possess other biological activities, such as anti-Gram-positive bacteria as SrtA transpeptidase inhibitors, ${ }^{5}$ anti-parasitic as cysteine protease inhibitors, ${ }^{6}$ and anti-virus as potent inhibitors of HIV-1 integrase. ${ }^{7}$

Since compounds with substitution of fluorine might have a higher stability against metabolic enzymes and a better membranous permeability, ${ }^{8,9}$ we therefore considered to introduce a trans-trifluoromethyl group to the $\alpha, \beta$-unsaturated vinylsulfone entity (Scheme 1). Thus, we initiated a program for the combinatorial synthesis of trifluoromethyl-substituted $(E)$ vinylsulfones and their biological evaluations as antitumor

${ }^{a}$ Obstetrics and Gynecology Hospital, Fudan University, 419 Fangxie Road, Shanghai 200011, China

${ }^{b}$ Department of Chemistry, Fudan University, 2005 Songhu Road, Shanghai 200433, China.E-mail:zhouw@fudan.edu.cn

${ }^{c}$ Department of Chemistry, Changsha University of Science and Technology, Changsha 410114, China

${ }^{d}$ School of Chemistry and Chemical Engineering, Hunan University of Science and Technology, Xiangtan 411201, China

$\dagger$ Electronic supplementary information (ESI) available. See DOI: 10.1039/c9ra06368d agents. Recently, we and others have involved in the synthesis of sulfonyl compounds, ${ }^{\mathbf{1 0 , 1 1}}$ and we identified that $(E)$ - $\beta$-trifluoromethyl vinylsulfones could be accessed through a threecomponent reaction of alkynes, Togni reagent, and sodium benzenesulfinates in DMSO under metal-free conditions at room temperature. ${ }^{12}$ We envisioned that the library of $(E)-\beta$ trifluoromethyl vinylsulfones could be constructed via this method through diversity-oriented synthesis.

Herein, we report the biological evaluation of a series of trans-trifluoromethyl vinylsulfone derivatives by aim to study the structure-activity relationship and identify a hit structure. By initial screening, we found that the introduction of $\beta$-transtrifluoromethyl group led to a potent activity against tumor cells proliferation.

\section{Results and discussion}

\section{Chemistry}

The synthetic route for the target $(E)$ - $\beta$-trifluoromethyl vinylsulfones was described in Scheme $2 .{ }^{12}$ By treatment of alkynes $\mathbf{1}$,

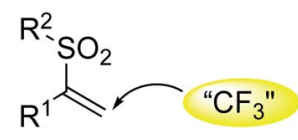

Scheme 1 Generation of (E)- $\beta$-trifluoromethyl vinylsulfones.<smiles>O=C1OI(C(F)(F)F)c2ccccc21</smiles>

Scheme 2 Synthesis of (E)- $\beta$-trifluoromethyl vinylsulfones. 
Table 1 In vitro antiproliferative activity of the target compounds

\begin{tabular}{|c|c|c|c|c|c|c|c|c|c|}
\hline & & $\begin{array}{c}\operatorname{Ar}^{1}= \\
\overline{1}\end{array}$ & II & DMs & & $\begin{array}{l}\mathrm{Ar}^{2} \\
3\end{array}$ & & & \\
\hline & $\mathrm{Ar}^{1}$ & $\mathrm{Ar}^{2}$ & \multicolumn{7}{|c|}{$\underline{\mathrm{IC}_{50} \text { values }(\mu \mathrm{M})}$} \\
\hline $3-1$ & & & 4.9 & 3.1 & 12.8 & 23.4 & 4.6 & 2.1 & 15.6 \\
\hline $3-2$ & & & 6.3 & 4.5 & 23.9 & 18.3 & 6.4 & 4.3 & 19.1 \\
\hline $3-3$ & & & 10.7 & 7.0 & $>25$ & 25.0 & 11.9 & $>25$ & $>25$ \\
\hline $3-4$ & & & 4.2 & 3.2 & 15.5 & $>25$ & 1.1 & 3.3 & $>25$ \\
\hline 3-5 & & & $>25$ & 23.7 & $>25$ & $>25$ & 12.9 & $>25$ & $>25$ \\
\hline $3-8$ & & & $>25$ & 9.1 & $>25$ & 16.6 & 3.6 & 19.5 & 12.4 \\
\hline $3-9$ & & & 19.0 & 4.7 & $>25$ & 6.3 & 4.9 & $>25$ & 12.2 \\
\hline $3-10$ & & & 3.8 & 2.4 & $>25$ & 14.2 & 2.9 & 9.7 & 22.5 \\
\hline $3-11$ & & & 5.6 & 3.7 & $>25$ & $>25$ & 8.3 & 15.1 & 18.4 \\
\hline $3-12$ & & & 8.9 & 2.3 & 16.8 & N.D & 6.6 & 14.8 & N.D \\
\hline
\end{tabular}




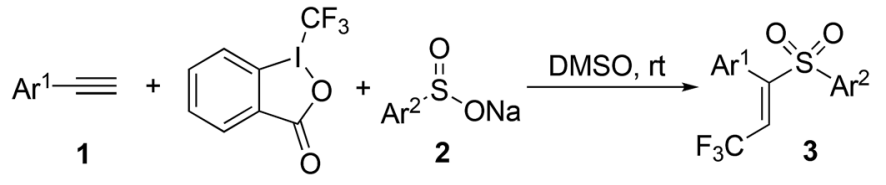

Doxorubicin

Togni reagent, and sodium benzenesulfinates 2 in DMSO under metal-free conditions at room temperature, a series of $(E)-\beta$ trifluoromethyl vinylsulfones 3 were obtained in moderate to good yields. On the basis of this strategy, the library of $(E)-\beta$ trifluoromethyl vinylsulfones was constructed easily with high efficiency.

\section{Biological activity}

We chose (E)-(3,3,3-trifluoro-1-(phenylsulfonyl)prop-1-en-1-yl) benzene as our structural template and modified the aryl groups. Subsequently, 15 derivatives were synthesized, which included various functional groups on both aryl groups $\left(\mathrm{Ar}^{1}\right.$ and $\mathrm{Ar}^{2}$ ) (Table 1, compounds 3-1 to 3-15). To our delight, these compounds had an excellent inhibitory activity against different tumor cells and especially against ES-2, HO-8910 and K562 with less than $10 \mu \mathrm{M}$ of $\mathrm{IC}_{50}$ values. As outlined in Table 1 , it was apparent that compounds 3-1 to 3-4 offered reasonable potency profiles when the electron-withdrawing groups were introduced to $\mathrm{Ar}^{1}$, while the electron-donating groups on $\mathrm{Ar}^{1}$ (compounds 35 and 6) would result in decreased activities. But the much stronger electron-withdrawing groups of cyano, fluoro, and trifluoromethyl didn't give better activity improvements (compounds 3-4, 3-7 to 3-9, and 3-11). A same electronic effect was also found on $\mathrm{Ar}^{2}$ (compounds 3-16 to 3-20), but the activity changes were much minor than that on $\mathrm{Ar}^{1}$.

To further validate the activities of electron-withdrawing groups, more substituents were introduced to investigate the structure-activity relations (Table 2, compounds 3-16 to 3-30). With the same results, the changes of $\mathrm{Ar}^{2}$ didn't make significant activity changes (compounds 3-16 to 3-20). Compound 327 , with an acetyl group on $p$-position of $\mathrm{Ar}^{1}$, all of the $\mathrm{IC}_{50}$ values on ES-2, HO-8910, A2780, and K562 decreased to nM level. However, the compound became more inactive when the acetyl group was connected on the $m$-position (compound 3-28). When the acetyl group was attached on $\mathrm{Ar}^{2}$, the activity was affected very little (compounds 3-20, 21, 23, and 25). Interestingly, when a strong electron-withdrawing carboxyl group was introduced to $\mathrm{Ar}^{1}$ (compounds 3-25 and 3-26), the activity was dramatically decreased. Therefore, it was reasonable to conclude that an acetyl group on the $p$-position of $\mathrm{Ar}^{1}$ was essential for the inhibitory activity, which might be due to a better noncovalent binding to the biological target by a moderate electronic withdrawing effect of the acetyl group.

Moreover, we synthesized compounds without double bond or trifluoromethyl group to identify the key structure of this skeleton (compounds 3-29 and 30). The results showed that the proliferation inhibition activities of these two compounds were both obviously inhibited compared with 3-27, about 40-, 11-, and 43-fold decrease for 3-29, and 60-, 16-, and 86-fold decrease for 3-30, on ES-2, HO-8190, and K562, respectively. Therefore, it showed that $\beta$-trifluoromethyl vinylsulfone along with an acetyl group on the $p$-position of $\mathrm{Ar}^{1}$ was essential for the antitumor activity. Subsequently, varies different electron-withdrawing groups were introduced to $\mathrm{Ar}^{2}$ with the structure of compound 3-27 as the structural template (Table 3, compounds 31-41). Unfortunately, no compound showed better profiles than that of compound 3-27.

The further time courses of the proliferation inhibitions on Skov3, HO-8910, ES-2, and K562 cells of compound 3-27 were conducted with different concentrations (10 and $1 \mu \mathrm{M})$ for different time periods $(12,24,36$, and $48 \mathrm{~h})$. As shown in Scheme 3, the cellular growth was obviously inhibited in a dose- 
Table 2 In vitro antiproliferative activity of the target compounds

\begin{tabular}{|c|c|c|c|c|c|c|c|c|c|}
\hline \multirow[b]{2}{*}{ Compounds } & \multirow[b]{2}{*}{$\mathrm{Ar}^{1}$} & \multirow[b]{2}{*}{$\mathrm{Ar}^{2}$} & \multicolumn{7}{|c|}{$\mathrm{IC}_{50}$ values $(\mu \mathrm{M})$} \\
\hline & & & ES-2 & HO-8910 & Skov3 & A2780 & K562 & A549 & Bel-7402 \\
\hline $3-16$ & & & 4.1 & 2.0 & 25.0 & 21.5 & 12.4 & 7.9 & $>25$ \\
\hline $3-18$ & & & 6.6 & 4.3 & $>25$ & 6.6 & 3.4 & 12.7 & 14.2 \\
\hline $3-20$ & & & 6.1 & 3.4 & 17.4 & 14.5 & 10.0 & $>25$ & $>25$ \\
\hline
\end{tabular}

3-21<smiles>Cc1ccc(C(=O)N2CCOCC2)cc1</smiles><smiles>CC(=O)c1ccc(C)cc1</smiles>

N.D $\quad 1.5$

$>25$

2.5

2.8

$6.0 \quad$ N.D<smiles>Cc1ccc(C(=O)N2CCOCC2)cc1</smiles><smiles>Cc1ccccc1</smiles>

$11.3 \quad 1.2$

17.9

9.2

1.4

8.9

$>25$

3-23<smiles>COC(=O)c1ccc(C)cc1</smiles><smiles>CC(=O)c1ccc(C)cc1</smiles>

16

1.9

$>25$

4.1

2.4

$15.7 \quad$ N.D

3-24<smiles>COC(=O)c1ccc(C)cc1</smiles><smiles>Cc1ccccc1</smiles>

$16.8 \quad 1.1$

12.2

9.8

1.4

5.9

$>25$

3-25<smiles>Cc1ccc(C(=O)O)cc1</smiles><smiles>CC(=O)c1ccc(C)cc1</smiles>

$>25$

7.1

$>25$

$>25$

6.0

N.D

3-26<smiles>Cc1ccc(C(=O)O)cc1</smiles><smiles>Cc1ccccc1</smiles>

$>25$

2.9

$>25$

14.9

4.4

$12.6>25$ 
Table 2 (Contd.)

Compounds

and time-dependent manner. All of tested cell lines were more sensitive to compound 3-27 treatment than doxorubicin.

Furthermore, special attention was paid to potential cell toxicities caused by the tested compounds. The cell toxicities of several active compounds were evaluated on human bone marrow mesenchymal stem cells (hBMSCs), compared with doxorubicin (Table 4). Except compound 3-31, all of these tested compounds had much lower toxicities on hBMSCs. A representative example, compound 3-27 had much higher inhibition activities on tumor cells with only $1 / 5$ toxicity on hBMSCs than doxorubicin, which indicates a potential development of such a new kind of skeleton as an alternative chemo drug.

\section{Conclusions}

In summary, we have identified several hits with a structural $\beta$ trans-trifluoromethyl vinylsulfone, which shows promising biological effects on antitumor with low cell toxicities, even the biological target is still unknown. The moderate electronwithdrawing groups, such as chloro, bromo, or acetyl group, on the $p$-position of $\mathrm{Ar}^{1}$ and with $\mathrm{Ar}^{2}$ unsubstituted both benefit the activity. More studies of structure-activity relationship, biological mechanism, and in vivo activity are likely to be subsequently conducted on such a lead compound.

\section{Experimental}

\section{Cell culture}

Human ovarian cancer cells (ES-2, HO-8910, Skov3, and A2780), human alveolar basal epithelial cells (A549), human hepatocellular carcinoma cells (Bel-7402), human myelogenous leukemia cells (K562) and human bone marrow mesenchymal stem cell (hBMSCs) were obtained from the Type Culture Collection of Chinese Academy of Sciences (Shanghai, China). Cancer cells were maintained in RPMI-1640 supplemented with antibiotics (100 units per $\mathrm{mL}$ penicillin $\mathrm{A}$ and $100 \mu \mathrm{g} \mathrm{mL}$ streptomycin) and $10 \% \mathrm{FBS}$ in an atmosphere of $5 \% \mathrm{CO}_{2}$ at $37^{\circ} \mathrm{C}$. hBMSCs was maintained in high glucose DMEM with $10 \%$ FBS. The medium was changed every three days. Exponentially growing cells were plated at a final concentration of 1 $\times 10^{4}$ cells per well in 96-well plates for cell proliferation assay.

\section{Cell viability}

The cell growth inhibitory effect of tested compounds determined using the MTT assay. After incubation for $24 \mathrm{~h}$ in 96-well 
Table 3 In vitro antiproliferative activity of the target compounds

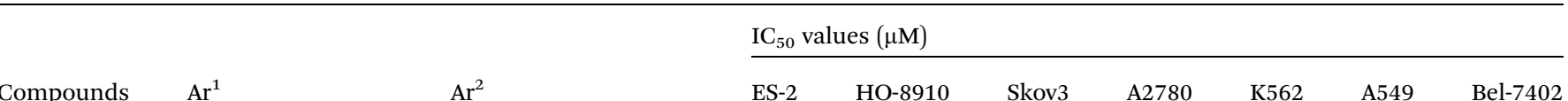

3-27<smiles>CC(=O)c1ccc(C)cc1</smiles>

$\mathrm{Ar}^{2}$<smiles>Cc1ccccc1</smiles>

$0.4 \quad 0.4$

5.7

0.8

0.2

$>25$

17.7

3-31<smiles>CC(=O)c1ccc(C)cc1</smiles><smiles>Cc1ccc(N)cc1</smiles>

$>25 \quad 9.9$

$>25$

14.6

5.9

$>25$

$>25$

3-32<smiles>CC(=O)c1ccc(C)cc1</smiles><smiles>CC(=O)c1ccc(C)cc1</smiles>

$4.5 \quad 4.8$

4.6 N.D

0.8

$5.3 \quad$ N.D<smiles>CC(=O)c1ccc(C)cc1</smiles><smiles>CC(=O)c1cccc(C)c1</smiles>

$13.1 \quad 1.5$

17.5

4.1

0.4

14.7

15.3

3-34<smiles>CC(=O)c1ccc(C)cc1</smiles><smiles>COC(=O)c1ccc(C)cc1</smiles>

$\begin{array}{ll}>25 & 7.7\end{array}$

$>25$

18.4

4.2

$>25$

$>25$

3-35<smiles>CC(=O)c1ccc(C)cc1</smiles><smiles>COS(=O)c1ccc(C)cc1</smiles>

$>25 \quad 2.2$

$>25$

15.5

6.5

20.2

$>25$<smiles>CC(=O)c1ccc(C)cc1</smiles><smiles>Cc1ccc([N+](=O)[O-])cc1</smiles>

$14.6 \quad 8.9$

$>25$

13.4

12.1

$13.6 \quad 20.2$

3-37<smiles>CC(=O)c1ccc(C)cc1</smiles><smiles>Cc1ccc(Cl)cc1</smiles>

$>25 \quad 2.0$

12.8

15.2

5.6

$>25$

13.6

3-38<smiles>CC(=O)c1ccc(C)cc1</smiles><smiles>Cc1ccc(C#N)cc1</smiles>

2

2.5

3.7

18.9

$>25$

$>25$

$>25$

3-39<smiles>CC(=O)c1ccc(C)cc1</smiles><smiles>Cc1ccc(F)cc1</smiles>

$6.5 \quad 4.2$

7.2

3.7

7.9

8.6

5.7

3-40<smiles>CC(=O)c1ccc(C)cc1</smiles><smiles>Cc1ccc(Br)cc1</smiles>

$3.2 \quad 2.2$

4.2

2.7

5.6

$>25$

13.5 
Table 3 (Contd.)

\begin{tabular}{|c|c|c|c|c|c|c|c|c|c|}
\hline \multirow[b]{2}{*}{ Compounds } & \multirow[b]{2}{*}{$\mathrm{Ar}^{1}$} & \multirow[b]{2}{*}{$\mathrm{Ar}^{2}$} & \multicolumn{7}{|c|}{$\mathrm{IC}_{50}$ values $(\mu \mathrm{M})$} \\
\hline & & & ES-2 & HO-8910 & Skov3 & A2780 & K562 & A549 & Bel-7402 \\
\hline $3-41$ & ? & & 2.3 & 3.1 & 4.8 & 12.6 & 9.2 & $>25$ & $>25$ \\
\hline Doxorubicin & & & 14.5 & 0.8 & 14.1 & 0.004 & 5.8 & $>25$ & 24.3 \\
\hline
\end{tabular}

plates, cells were treated with various concentrations $(25,12.5$, $6.25,3.12,1.56,0.78,0.39$, and $0.20 \mu \mathrm{M})$ of tested compounds for 48 hours, and the MTT solution $\left(0.5 \mathrm{mg} \mathrm{mL}^{-1}\right)$ was added. After $4 \mathrm{~h}$ of incubation at $37^{\circ} \mathrm{C}$ for MTT-formazan formation, the supernatant was removed and $100 \mu \mathrm{L}$ of dimethyl sulfoxide (DMSO) was added into each well. Absorbance at $490 \mathrm{~nm}$ was determined spectrophotometrically by using a microplate reader (Epoch, BioTek Instruments, Inc., VT, USA). Each concentration was performed in triplicate. Antitumor activity was evaluated using $\mathrm{IC}_{50}$ determined by non-linear regression analysis.

\section{General experimental procedure for the synthesis of $(E)-\beta$ - trifluoromethyl vinylsulfones from alkyne, Togni reagent, and sodium benzenesulfinate}

Under nitrogen atmosphere, a mixture of alkyne $\mathbf{1}(0.2 \mathrm{mmol})$ and Togni reagent $(0.22 \mathrm{mmol})$ in DMSO $(1.0 \mathrm{~mL})$ was stirred for several minutes. Then sodium benzenesulfinate $2(0.4 \mathrm{mmol})$ in DMSO $(2.0 \mathrm{~mL})$ was added to the solution. The mixture was stirred overnight at room temperature. After completion of reaction as indicated by TLC, water $(10 \mathrm{~mL})$ was added and the mixture was extracted by EtOAc $(3 \times 10 \mathrm{~mL})$. The solvent was evaporated and the residue was purified by flash column chromatography (EtOAc/n-hexane, $1: 8$ ) to give the desired product 3. Data of typical examples are shown below.

(E)-1-Bromo-4-(3,3,3-trifluoro-1-(phenylsulfonyl)prop-1-en-1yl)benzene (3-1). White solid, $59.4 \mathrm{mg}, 76.3 \%$ yield, ${ }^{1} \mathrm{H}$ NMR $\left(400 \mathrm{MHz}, \mathrm{CDCl}_{3}\right) \delta$ 7.67-7.54 (m, 3H), 7.50-7.37 (m, 4H), 7.17 (q, $J=7.1 \mathrm{~Hz}, 1 \mathrm{H}), 6.91-6.82(\mathrm{~m}, 2 \mathrm{H}) ;{ }^{19} \mathrm{~F}$ NMR $(376 \mathrm{MHz}$, $\left.\mathrm{CDCl}_{3}\right) \delta-58.2(\mathrm{~d}, J=7.1 \mathrm{~Hz}) ;{ }^{13} \mathrm{C}$ NMR $\left(100 \mathrm{MHz}, \mathrm{CDCl}_{3}\right)$ $\delta 152.2(\mathrm{~d}, J=4.8 \mathrm{~Hz}), 136.0,134.5,131.4,131.1(\mathrm{~d}, J=1.3 \mathrm{~Hz})$, 129.2, 129.1, 126.7, 125.8 (q, $J=36.0 \mathrm{~Hz}), 124.9,121.4(\mathrm{q}, J=$ $273.5 \mathrm{~Hz}$ ); LC-MS: $>97 \%$ purity; HRMS (ESI) calcd for $\mathrm{C}_{15} \mathrm{H}_{11}$ $\mathrm{BrF}_{3} \mathrm{O}_{2} \mathrm{~S}: 390.9610\left(\mathrm{M}+\mathrm{H}^{+}\right)$, found: 390.9634 .

(E)-1-Chloro-4-(3,3,3-trifluoro-1-(phenylsulfonyl)prop-1-en-1yl)benzene (3-2). White solid, $51.4 \mathrm{mg}, 74.3 \%$ yield, ${ }^{1} \mathrm{H}$ NMR $\left(400 \mathrm{MHz}, \mathrm{CDCl}_{3}\right) \delta 7.62(\mathrm{t}, J=7.4 \mathrm{~Hz}, 1 \mathrm{H}), 7.61-7.59(\mathrm{~m}, 2 \mathrm{H})$, 7.49-7.42 (m, 2H), $7.25(\mathrm{~d}, J=8.6 \mathrm{~Hz}, 2 \mathrm{H}), 7.17(\mathrm{q}, J=7.1 \mathrm{~Hz}$, $1 \mathrm{H}), 6.94(\mathrm{~d}, J=8.5 \mathrm{~Hz}, 2 \mathrm{H}) ;{ }^{19} \mathrm{~F} \mathrm{NMR}\left(376 \mathrm{MHz}, \mathrm{CDCl}_{3}\right) \delta-58.3$ $(\mathrm{d}, J=7.0 \mathrm{~Hz}) ;{ }^{13} \mathrm{C}$ NMR $\left(100 \mathrm{MHz}, \mathrm{CDCl}_{3}\right) \delta 152.2,136.5,136.0$, 134.5, 130.9 (d, $J=1.2 \mathrm{~Hz}), 129.2,129.1,128.4,126.2,125.8$ (q, $J$ $=36.0 \mathrm{~Hz}$ ), 121.4 (q, $J=273.6 \mathrm{~Hz})$; LC-MS: >97\% purity; HRMS (ESI) calcd for $\mathrm{C}_{15} \mathrm{H}_{11} \mathrm{ClF}_{3} \mathrm{O}_{2} \mathrm{~S}$ : $347.0115\left(\mathrm{M}+\mathrm{H}^{+}\right)$, found: 347.0104.
(E)-1-(3,3,3-Trifluoro-1-(phenylsulfonyl)prop-1-en-1-yl)-4(trifluoromethoxy)benzene (3-3). White solid, $50.0 \mathrm{mg}, 63.1 \%$ yield, ${ }^{1} \mathrm{H}$ NMR $\left(400 \mathrm{MHz}, \mathrm{CDCl}_{3}\right) \delta 7.62(\mathrm{t}, J=7.4 \mathrm{~Hz}, 1 \mathrm{H}), 7.58^{-}$ $7.55(\mathrm{~m}, 2 \mathrm{H}), 7.45(\mathrm{t}, J=7.8 \mathrm{~Hz}, 2 \mathrm{H}), 7.18(\mathrm{q}, J=7.1 \mathrm{~Hz}, 1 \mathrm{H}), 7.12$ $(\mathrm{d}, J=8.2 \mathrm{~Hz}, 2 \mathrm{H}), 7.04(\mathrm{~d}, J=8.8 \mathrm{~Hz}, 2 \mathrm{H}) ;{ }^{19} \mathrm{~F}$ NMR $(376 \mathrm{MHz}$, $\left.\mathrm{CDCl}_{3}\right) \delta-57.9(\mathrm{~s}, 3 \mathrm{~F}),-58.4(\mathrm{~d}, J=7.0 \mathrm{~Hz}, 3 \mathrm{~F}) ;{ }^{13} \mathrm{C}$ NMR $(100$ $\left.\mathrm{MHz}, \mathrm{CDCl}_{3}\right) \delta 152.0(\mathrm{~d}, J=4.9 \mathrm{~Hz}), 150.4,135.9,134.5,131.3(\mathrm{~d}$, $J=1.0 \mathrm{~Hz}), 129.2,129.1,126.3,125.8(\mathrm{q}, J=36.0 \mathrm{~Hz}), 121.3(\mathrm{q}, J$ $=267.2 \mathrm{~Hz}), 120.2(\mathrm{q}, J=258.4 \mathrm{~Hz}), 120.1$; LC-MS: >97\% purity; HRMS (ESI) calcd for $\mathrm{C}_{16} \mathrm{H}_{11} \mathrm{~F}_{6} \mathrm{O}_{3} \mathrm{~S}: 397.0328\left(\mathrm{M}+\mathrm{H}^{+}\right)$, found: 397.0314 .

(E)-1-(3,3,3-Trifluoro-1-(phenylsulfonyl)prop-1-en-1-yl)-4(trifluoromethyl)benzene (3-4). White solid, $53.5 \mathrm{mg}, 70.4 \%$ yield, ${ }^{1} \mathrm{H}$ NMR $\left(400 \mathrm{MHz}, \mathrm{CDCl}_{3}\right) \delta 7.66(\mathrm{~d}, J=7.0 \mathrm{~Hz}, 1 \mathrm{H}), 7.63-$ $7.52(\mathrm{~m}, 4 \mathrm{H}), 7.51-7.48(\mathrm{~m}, 2 \mathrm{H}), 7.29-7.22(\mathrm{~m}, 1 \mathrm{H}), 7.14(\mathrm{~d}, J=$ $7.3 \mathrm{~Hz}, 2 \mathrm{H}) ;{ }^{19} \mathrm{~F} \mathrm{NMR}\left(376 \mathrm{MHz}, \mathrm{CDCl}_{3}\right) \delta-58.3(\mathrm{~d}, J=6.8 \mathrm{~Hz})$, $-63.0(\mathrm{~s}) ;{ }^{13} \mathrm{C}$ NMR $\left(100 \mathrm{MHz}, \mathrm{CDCl}_{3}\right) \delta 152.0,135.8,134.7$, 131.9, 131.6, 130.1, 129.3, 129.1, 126.1 (q, $J=36.3 \mathrm{~Hz}), 125.0$, 121.3 (q, $J=273.2 \mathrm{~Hz}$ ); LC-MS: $>97 \%$ purity; HRMS (ESI) calcd for $\mathrm{C}_{16} \mathrm{H}_{11} \mathrm{~F}_{6} \mathrm{O}_{2} \mathrm{~S}: 381.0378\left(\mathrm{M}+\mathrm{H}^{+}\right)$, found: 381.0369.

(E)-4-(3,3,3-Trifluoro-1-(phenylsulfonyl)prop-1-en-1-yl)-1,1'biphenyl (3-5). Pale yellow solid, $55.0 \mathrm{mg}, 70.9 \%$ yield, ${ }^{1} \mathrm{H}$ NMR
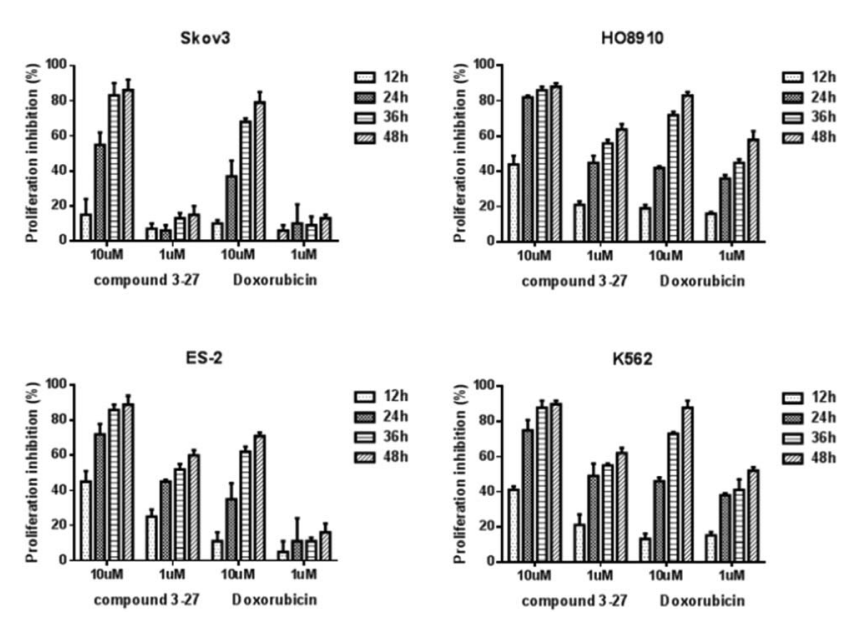

Scheme 3 Dose- and time-dependent effect of compound 3-27 on cancer cells (Skov3, HO-8910, ES-2, and K562). Cell proliferation inhibitions were examined by the MTT method as described in Experimental. The data has been plotted using means S.E. of triplicate determinations. 
Table 4 Cell toxicities of the selected compounds on hBMSCs

\begin{tabular}{lllllll}
\hline Compounds & $3-27$ & $3-32$ & $3-36$ & $3-37$ & $3-39$ & $3-40$ \\
$\mathrm{IC}_{50}(\mu \mathrm{M})$ & $5.4 \pm 0.61$ & $0.65 \pm 0.45$ & $15.3 \pm 0.72$ & $6.5 \pm 0.56$ & $6.8 \pm 1.57$ & $8.4 \pm 0.91$
\end{tabular}

$\left(400 \mathrm{MHz}, \mathrm{CDCl}_{3}\right) \delta 7.67-7.56(\mathrm{~m}, 5 \mathrm{H}), 7.53(\mathrm{~d}, J=8.2 \mathrm{~Hz}, 2 \mathrm{H})$, $7.50-7.42(\mathrm{~m}, 4 \mathrm{H}), 7.40(\mathrm{~d}, J=7.2 \mathrm{~Hz}, 1 \mathrm{H}), 7.22(\mathrm{q}, J=7.1 \mathrm{~Hz}, 1 \mathrm{H})$, $7.10(\mathrm{~d}, J=8.2 \mathrm{~Hz}, 2 \mathrm{H}) ;{ }^{19} \mathrm{~F} \mathrm{NMR}\left(376 \mathrm{MHz}, \mathrm{CDCl}_{3}\right) \delta-58.1(\mathrm{~d}, J=$ $7.1 \mathrm{~Hz}) ;{ }^{13} \mathrm{C}$ NMR $\left(100 \mathrm{MHz}, \mathrm{CDCl}_{3}\right) \delta 153.1,142.7,139.7,136.3$, 134.3, 130.03, 129.1, 128.9, 128.0, 127.0, 126.6, 125.3 (q, $J=35.9$ $\mathrm{Hz}), 124.8,121.6$ (q, $J=284.1 \mathrm{~Hz}$ ); LC-MS: >97\% purity; HRMS (ESI) calcd for $\mathrm{C}_{21} \mathrm{H}_{16} \mathrm{~F}_{3} \mathrm{O}_{2} \mathrm{~S}: 389.0818\left(\mathrm{M}+\mathrm{H}^{+}\right)$, found: 389.0803 .

(E)-1-(3,3,3-Trifluoro-1-(phenylsulfonyl)prop-1-en-1-yl) naphthalene (3-6). Yellow oil, $32.3 \mathrm{mg}, 44.6 \%$ yield, ${ }^{1} \mathrm{H}$ NMR $\left(400 \mathrm{MHz}, \mathrm{CDCl}_{3}\right) \delta 7.88(\mathrm{~d}, J=8.3 \mathrm{~Hz}, 1 \mathrm{H}), 7.79(\mathrm{~d}, J=8.2 \mathrm{~Hz}$, 1H), 7.51-7.46 (m, 3H), $7.44(\mathrm{~d}, J=7.0 \mathrm{~Hz}, 1 \mathrm{H}), 7.42-7.39(\mathrm{~m}$, 1H), 7.38-7.33 (m, 2H), 7.32-7.27 (m, 3H), $7.03(\mathrm{~d}, J=7.1,1 \mathrm{H})$; ${ }^{19} \mathrm{~F} \mathrm{NMR}\left(376 \mathrm{MHz}, \mathrm{CDCl}_{3}\right) \delta-60.1(\mathrm{~d}, J=7.0 \mathrm{~Hz}) ;{ }^{13} \mathrm{C}$ NMR $(100$ $\left.\mathrm{MHz}, \mathrm{CDCl}_{3}\right) \delta 152.0,134.3,132.9,131.4,130.6,129.7,129.4$, 129.2, 128.9, 128.6, 128.1, 127.2 (q, $J=35.9 \mathrm{~Hz}$ ), 126.7, 126.2, 124.6, 124.3, 121.4 (q, $J=273.6 \mathrm{~Hz}$ ); LC-MS: >97\% purity; HRMS (ESI) calcd for $\mathrm{C}_{19} \mathrm{H}_{14} \mathrm{~F}_{3} \mathrm{O}_{2} \mathrm{~S}: 363.0661\left(\mathrm{M}+\mathrm{H}^{+}\right)$, found: 363.0659 .

(E)-1-Chloro-4-((1-(4-chlorophenyl)-3,3,3-trifluoroprop-1-en1-yl)sulfonyl)benzene (3-12). White solid, $34.0 \mathrm{mg}, 44.8 \%$ yield, ${ }^{1} \mathrm{H}$ NMR (400 MHz, $\mathrm{CDCl}_{3}$ ) $\delta 7.53-7.45(\mathrm{~m}, 4 \mathrm{H}), 7.33-7.28(\mathrm{~m}, 2 \mathrm{H})$, 7.23-7.13 (m, 1H), 7.05-6.89 (m, 2H); ${ }^{19} \mathrm{~F}$ NMR (376 MHz, $\mathrm{CDCl}_{3}$ ) $\delta-58.3(\mathrm{~d}, J=6.8 \mathrm{~Hz}) ;{ }^{13} \mathrm{C} \mathrm{NMR}\left(100 \mathrm{MHz}, \mathrm{CDCl}_{3}\right) \delta 151.8,141.5$, 136.8, 134.6, 130.9, 130.5, 129.6, 128.6, $126.2(\mathrm{q}, J=36.2 \mathrm{~Hz})$, 126.0, 121.3 (q, $J=274.1 \mathrm{~Hz}$ ); LC-MS: >97\% purity; HRMS (ESI) calcd for $\mathrm{C}_{15} \mathrm{H}_{10} \mathrm{Cl}_{2} \mathrm{~F}_{3} \mathrm{O}_{2} \mathrm{~S}: 380.9725\left(\mathrm{M}+\mathrm{H}^{+}\right)$, found: 380.9708 .

(E)-1-(4-(3,3,3-Trifluoro-1-(phenylsulfonyl)prop-1-en-1-yl) phenyl)ethanone (3-27). White solid, $56.6 \mathrm{mg}, 79.9 \%$ yield, ${ }^{1} \mathrm{H}$ NMR (400 MHz, $\left.\mathrm{CDCl}_{3}\right) \delta 7.85(\mathrm{~d}, J=8.5 \mathrm{~Hz}, 2 \mathrm{H}), 7.63(\mathrm{t}, J=$ $7.3 \mathrm{~Hz}, 1 \mathrm{H}), 7.58-7.55(\mathrm{~m}, 2 \mathrm{H}), 7.45$ (t, $J=7.8 \mathrm{~Hz}, 2 \mathrm{H}), 7.19$ (q, $J$ $=7.1 \mathrm{~Hz}, 1 \mathrm{H}), 7.10(\mathrm{~d}, J=8.3 \mathrm{~Hz}, 2 \mathrm{H}), 2.59(\mathrm{~s}, 3 \mathrm{H}) ;{ }^{19} \mathrm{~F} \mathrm{NMR}(376$ $\left.\mathrm{MHz}, \mathrm{CDCl}_{3}\right) \delta-58.3(\mathrm{~d}, J=7.2 \mathrm{~Hz}) ;{ }^{13} \mathrm{C} \mathrm{NMR}\left(100 \mathrm{MHz}, \mathrm{CDCl}_{3}\right)$ $\delta$ 197.2, 152.3, 137.9, 135.9, 134.6, 132.5, 129.9, 129.3, 129.1, 127.8, 125.8 (q, $J=36.1 \mathrm{~Hz}), 121.3$ (q, $J=273.5 \mathrm{~Hz}), 26.6$; LC-MS: $>97 \%$ purity; HRMS (ESI) calcd for $\mathrm{C}_{17} \mathrm{H}_{14} \mathrm{~F}_{3} \mathrm{O}_{3} \mathrm{~S}: 355.0610(\mathrm{M}+$ $\mathrm{H}^{+}$), found: 355.0617 .

\section{Conflicts of interest}

There are no conflicts to declare.

\section{Acknowledgements}

Financial supports from the Natural Science Foundation of Science and Technology Commission of Shanghai Municipality (No. 19ZR1407100) and Double First-Class Construct Project of Fudan University (No. IDH1615098) are gratefully acknowledged.

\section{Notes and references}

1 (a) X. Dneg, H. Cao, C. Chen, H. Zhou and L. Yu, Sci. Bull., 2019, 64, 1280; (b) M. Liu, Y. Li, L. Yu, Q. Xu and X. Jiang,
Sci. China: Chem., 2018, 61, 294; (c) T. Prochnow, A. Maroneze, D. F. Back and G. Zeni, J. Org. Chem., 2019, 84, 2891; (d) L.-H. Lu, S.-J. Zhou, W.-B. He, W. Xia, P. Chen, X. Yu, X. Xu and W.-M. He, Org. Biomol. Chem., 2018, 16, 9064; (e) S. Kodama, T. Saeki, K. Mihara, S. Higashimae, S.-i. Kawaguchi, M. Sonoda, A. Nomoto and A. Ogawa, J. Org. Chem., 2017, 82, 12477; $(f)$ C. Ma, J.-Y. Zhou, Y.-Z. Zhang, Y. Jiao, G.-J. Mei and F. Shi, Chem.Asian J., 2018, 13, 2549; (g) C. Ma, F. Jiang, F.-T. Sheng, Y. Jiao, G.-J. Mei and F. Shi, Angew. Chem., Int. Ed., 2019, 58, 3014; (h) X. Gong, J. Chen, X. Li, W. Xie and J. Wu, Chem.-Asian J., 2018, 13, 2543; (i) D. Ren, B. Liu, X. Li, S. Koniarz, M. Pawlicki and P. J. Chmielewski, Org. Chem. Front., 2019, 6, 908; (j) Z. Wang, L. Yang, H.-L. Liu, W.-H. Bao, Y.-Z. Tan, M. Wang, Z. Tang and W.-M. He, Youji Huaxue, 2018, 38, 2639; (k) Y. Zheng, M. Liu, G. Qiu, W. Xie and J. Wu, Tetrahedron, 2019, 75, 1663.

2 S. Woo, J. Kim, M. Moon, S. Han, S. Yeon, J. Choi, B. Jang, H. Song, Y. Kang, J. Kim, J. Lee, D. Kim, O. Hwang and K. Park, J. Med. Chem., 2014, 57, 1473.

3 J. A. Lee, J. H. Kim, S. Y. Woo, H. J. Son, S. H. Han, B. K. Jang, J. W. Choi, D. J. Kim, K. D. Park and O. Hwang, Br. J. Pharmacol., 2015, 172, 1087.

4 (a) L. Ni, S. Zheng, P. K. Somers, L. K. Hoong, R. R. Hill, E. M. Marino, K.-L. Suen, U. Saxena and C. Q. Meng, Bioorg. Med. Chem. Lett., 2003, 13, 745; (b) W. Xie, Y. Wu, J. Zhang, Q. Mei, Y. Zhang, N. Zhu, R. Liu and H. Zhang, Eur. J. Med. Chem., 2018, 145, 35; (c) W. Xie, S. Xie, Y. Zhou, X. Tang, J. Liu, W. Yang and M. Qiu, Eur. J. Med. Chem., 2014, 81, 22; (d) W. Xie, H. Zhang, J. He, J. Zhang, Q. Yu, C. Luo and S. Li, Bioorg. Med. Chem. Lett., 2017, 27, 530; (e) T. Guo, Y. Liu, Y.-H. Zhao, P.-K. Zhang, S.-L. Han and H.-M. Liu, Tetrahedron Lett., 2016, 57, 3920.

5 (a) B. A. Frankel, M. Bentley, R. G. Kruger and D. G. Mccafferty, J. Am. Chem. Soc., 2004, 126, 3404; (b) D. Chen, Y. Shan, J. Li, J. You, X. Sun and G. Qiu, Org. Lett., 2019, 21, 4044; (c) Y. Zheng, M. Liu, G. Qiu, W. Xie and J. Wu, Tetrahedron, 2019, 75, 1663; (d) R. Liu, W. Xie, H. Zhou, Y. Zhang and G. Qiu, J. Org. Chem., 2019, 84(18), 11763-11773; (e) Y.-H. Wang, B. Ouyang, G. Qiu, H. Zhou and J.-B. Liu, Org. Biomol. Chem., 2019, 17, 4335; (f) G. Qiu, Z. Chen, W. Xie and H. Zhou, Eur. J. Org. Chem., 2019, 4327; (g) Y.-C. Wang, R.-X. Wang, G. Qiu, H. Zhou, W. Xie and J.-B. Liu, Org. Chem. Front., 2019, 6, 2471; (h) Y.-H. Wang, G. Qiu, H. Zhou, W. Xie and J.-B. Liu, Tetrahedron, 2019, 75, 3850; ( $i$ ) R.-X. Wang, Z. Fang, G. Qiu, W. Xie and J.-B. Liu, Synthesis, 2019, DOI: 10.1055/s-00391690155.

6 I. D. Kerr, J. H. Lee, C. J. Farady, R. Marion, M. Rickert, M. Sajid, K. C. Pandey, C. R. Caffrey, J. Legac, E. Hansell, 
J. McKerrow, C. S. Craik, P. J. Rosenthal and L. S. Brinen, J. Biol. Chem., 2009, 284, 25697.

7 D. C. Meadows and J. Gervay-Hague, Med. Res. Rev., 2006, 26, 793.

8 (a) S. Purser, P. R. Moore, S. Swallow and V. Gouverneur, Chem. Soc. Rev., 2008, 37, 320; (b) D. O'Hagan, Chem. Soc. Rev., 2008, 37, 308; (c) W. K. Hagmann, J. Med. Chem., 2008, 51, 4359; (d) J. Wang, M. SanchezRosello, J. L. Aceña, C. del Pozo, A. E. Sorochinsky, S. V. Fustero, A. Soloshonok and H. Liu, Chem. Rev., 2014, 114, 2432; (e) Y. Zhou, J. Wang, Z. Gu, S. Wang, W. Zhu, J. L. Aceña, V. A. Soloshonok, K. Izawa and H. Liu, Chem. Rev., 2016, 116, 422.

9 (a) X. Fu, Y. Meng, X. Li, M. Stepień and P. J. Chmielewski, Chem. Commun., 2018, 54, 2510; (b) X. Li, Y. Meng, P. Yi, M. Stepień and P. J. Chmielewski, Angew. Chem., Int. Ed., 2017, 56, 10810; (c) B. Liu, T. Yoshida, X. Li, M. Stepień, H. Shinokubo and P. J. Chmielewski, Angew. Chem., Int. Ed., 2016, 55, 13142; (d) Z. Deng, X. Li, M. Stepień and P. J. Chmielewski, Chem. -Eur. J., 2016, 22, 4231; (e) K. Deng, X. Li and H. Huang, Electrochim. Acta, 2016, 204, 84; (f) B. Liu, X. Li, M. Stepień and P. J. Chmielewski, Chem. -Eur. J., 2015, 21, 7790; $(g)$ B. Liu, H. Fang, X. Li, W. Cai, L. Bao, M. Rudolf, F. Plass, L. Fan, X. Lu and D. M. Guldi, Chem. -Eur. J., 2015, 21, 746; (h) B. Liu, X. Li, J. Maciołek, M. Stepień and P. J. Chmielewski, J. Org. Chem., 2014, 79, 3129; (i) B. Liu, X. Li, X. Xu, M. Stepień and P. J. Chmielewski, J. Org. Chem., 2013, 78, 1354; (j) X. Li, B. Liu, P. J. Chmielewski and X. Xu, J. Org. Chem., 2012, 77, 8206; (k) X. Li, B. Liu, X. Yu, P. Yi, R. Yi and P. J. Chmielewski, J. Org. Chem., 2012, 77, 2431; (l) X. Li, B. Liu, P. Yi, R. Yi, X. Yu and P. J. Chmielewski, J. Org. Chem., 2011, 76, 2345.

10 For recent examples, see:(a) X. Gong, M. Wang, S. Ye and J. Wu, Org. Lett., 2019, 21, 1156; (b) X. Wang, M. Yang, W. Xie, X. Fan and J. Wu, Chem. Commun., 2019, 55, 6010; (c) S. Ye, D. Zheng, J. Wu and G. Qiu, Chem. Commun., 2019, 55, 2214; (d) S. Ye, Y. Li, J. Wu and Z. Li, Chem.
Commun., 2019, 55, 2489; (e) X. Gong, X. Li, W. Xie, J. Wu and S. Ye, Org. Chem. Front., 2019, 6, 1863; $(f)$ J. Zhang, W. Xie, S. Ye and J. Wu, Org. Chem. Front., 2019, 6, 2254; (g) S. Ye, T. Xiang, X. Li and J. Wu, Org. Chem. Front., 2019, 6, 2183; (h) S. Ye, X. Li, W. Xie and J. Wu, Asian J. Org. Chem., 2019, 8, 893; (i) S. Ye, X. Li, W. Xie and J. Wu, Eur. J. Org. Chem., 2019, DOI: 10.1002/ejoc.201900396; (j) J. Zhang, X. Li, W. Xie, S. Ye and J. Wu, Org. Lett., 2019, 21, 4950; (k) Y. Zong, Y. Lang, M. Yang, X. Li, X. Fan and J. Wu, Org. Lett., 2019, 21, 1935; (l) F.-S. He, Y. Wu, X. Li, H. Xia and J. Wu, Org. Chem. Front., 2019, 6, 1873.

11 (a) C. Wu, L.-H. Lu, A.-Z. Peng, G.-K. Jia, C. Peng, Z. Cao, Z. Tang, W.-M. He and X. Xu, Green Chem., 2018, 20, 3683; (b) L.-H. Lu, S.-J. Zhou, M. Sun, J.-L. Chen, W. Xia, X. Yu, X. Xu and W.-M. He, ACS Sustainable Chem. Eng., 2019, 7, 1574; (c) K.-J. Liu, S. Jiang, L.-H. Lu, L.-L. Tang, S.-S. Tang, H.-S. Tang, Z. Tang, W.-M. He and X. Xu, Green Chem., 2018, 20, 3038; (d) L.-Y. Xie, S. Peng, J.-X. Tan, R.-X. Sun, X. Yu, N.-N. Dai, Z.-L. Tang, X. Xu and W.-M. He, ACS Sustainable Chem. Eng., 2018, 6, 16976; (e) L.-Y. Xie, S. Peng, F. Liu, J.-Y. Yi, M. Wang, Z. Tang, X. Xu and W.-M. He, Adv. Synth. Catal., 2018, 360, 4259; (f) L.-Y. Xie, S. Peng, L.-H. Lu, J. Hu, W.-H. Bao, F. Zeng, Z. Tang, X. Xu and W.-M. He, ACS Sustainable Chem. Eng., 2018, 6, 7989; (g) C. Wu, H.-J. Xiao, S.-W. Wang, M.-S. Tang, Z.-L. Tang, W. Xia, W.-F. Li, C. Zhong and W.-M. He, ACS Sustainable Chem. Eng., 2019, 7, 2169; (h) L.-Y. Xie, S. Peng, L.-L. Jiang, X. Peng, W. Xia, X. Yu, X.-X. Wang, Z. Cao and W.-M. He, Org. Chem. Front., 2019, 6, 167; (i) L.-Y. Xie, S. Peng, F. Liu, G.-R. Chen, W. Xia, X. Yu, W.-F. Li, Z. Cao and W.-M. He, Org. Chem. Front., 2018, 5, 2604; (j) C. Wu, Z. Wang, Z. Hu, F. Zeng, X.-Y. Zhang, Z. Cao, Z. Tang, W.-M. He and X. Xu, Org. Biomol. Chem., 2018, 16, 3177; (k) C. Wu, J. Wang, X.-Y. Zhang, G.-K. Jia, Z. Cao, Z. Tang, X. Yu, X. Xu and W.-M. He, Org. Biomol. Chem., 2018, 16, 5050.

12 Y. Xiang, Y. Li, Y. Kuang and J. Wu, Adv. Synth. Catal., 2017, 359, 2605. 